\title{
O alagamento do solo afeta a sobrevivência, o crescimento e o metabolismo de Aegiphila sellowiana Cham. (Lamiaceae)?
}

\section{Soil flooding affects the survival, growth and metabolism of Aegiphila sellowiana Cham. (Lamiaceae)?}

\author{
Cristiano Medri ${ }^{1}$; José Antônio Pimenta²; Eduardo Augusto Ruas ${ }^{3}$; Luiz Antônio \\ de Souza ${ }^{4}$; Paulo Souza Medri ${ }^{5}$; Sandra Sayhun ${ }^{6}$; Edmilson Bianchini ${ }^{7}$; Moacyr \\ Eurípedes Medri ${ }^{8}$
}

\section{Resumo}

\begin{abstract}
Visando a elucidar aspectos da tolerância à hipoxia, plantas de Aegiphila sellowiana Cham. (Lamiaceae) foram submetidas a diferentes períodos de inundação. A sobrevivência, o crescimento, a condutância estomática e a fotossíntese foram avaliados. Houve mortalidade de 33,3\% das plantas alagadas. Dentre as $66,7 \%$ sobreviventes, a taxa de crescimento relativo (TCR) de raízes, caule, folhas e da planta inteira foi inferior à TCR das plantas cultivadas em solo drenado. As plantas alagadas apresentaram produção de raízes diageotrópicas, maior abscisão foliar e baixa produção de novas folhas. $\mathrm{O}$ alagamento provocou redução na condutância estomática e na taxa fotossintética. Sugere-se que A. sellowiana possui intolerância ao alagamento do solo.
\end{abstract}

Palavras-Chave: Tolerância ao alagamento. Hipoxia. TCR. Condutância estomática. Fotossíntese.

1 Universidade estadual do Norte do Paraná (UENP), Professor adjunto da Universidade Estadual do Norte do Paraná (UENP), Campus Luiz Meneghel, Centro de Ciências Biológicas, Setor de Biologia. Contato principal para correspondência: cmedri@ uenp.edu.br.

2 Universidade Estadual de Londrina (UEL), Professor associado da Universidade Estadual de Londrina (UEL), Centro de Ciências Biológicas, Departamento de Biologia Animal e Vegetal. E-mail: pimenta@uel.br.

3 Universidade Estadual de Londrina (UEL), Pós-doutorando na Universidade Estadual de Londrina (UEL), Centro de Ciências Biológicas, Departamento de Biologia Geral. E-mail: edu_wicca@yahoo.com.br.

4 Universidade Estadual de Maringá (UEM), Professor titular da Universidade Estadual de Maringá (UEM), Centro de Ciências Biológicas, Departamento de Biologia. E-mail: lasouza@uem.br.

5 Universidade Estadual de Londrina, Mestre pela Universidade Estadual de Londrina, Centro de Ciências Biológicas, Departamento de Biologia Animal e Vegetal. E-mail: paulomedri@hotmail.com.

6 Universidade Estadual de Londrina (UEL), Mestre pela Universidade Estadual de Londrina, Centro de Ciências Biológicas, Departamento de Biologia Geral. E-mail: sandra_biopr@yahoo.com.br.

7 Universidade Estadual de Londrina (UEL), Professor associado da Universidade Estadual de Londrina (UEL), Centro de Ciências Biológicas, Departamento de Biologia Animal e Vegetal. E-mail: bianchi@uel.br.

8 Universidade Estadual de Londrina (UEL), Professor associado da Universidade Estadual de Londrina (UEL), Centro de Ciências Biológicas, Departamento de Biologia Animal e Vegetal. E-mail: moacyrmedri@uel.br. 


\begin{abstract}
In order to elucidate aspects of tolerance to hypoxia, Aegiphila sellowiana Cham. (Lamiaceae) plants were submitted to different periods of flooding. The survival, growth, stomatal conductance and photosynthesis were evaluated. There was $33,3 \%$ of mortality of flooded plants. Among the $66,7 \%$ of survivors, the relative growth rate (RGR) of roots, stems, leaves and whole plant was lower than the RGR of plants cultivated in drained soil. Flooded plants presented diageotrophic root production, increased leaf abscission and low production of new leaves. Flooding caused a reduction in stomatal conductance and photosynthetic rate. It is suggested that A. sellowiana has intolerance to soil flooding.

Keywords: Flooding tolerance. Hypoxia. RGR. Stomatal conductance. Photosynthesis.
\end{abstract}

\section{Introdução}

A maioria das comunidades vegetais naturalmente inundáveis ocorre nas regiões tropicais. Estima-se que, somente na América do Sul, haja em torno de dois milhões de $\mathrm{Km}^{2}$ de áreas inundáveis, o que representa, aproximadamente, $11 \%$ de toda a área desta vasta região (JUNK, 1993). A deficiência de oxigênio e o baixo potencial redox do solo, induzidos pelo alagamento, afetam desfavoravelmente vários aspectos da fisiologia vegetal, como mudanças na assimilação de carbono, absorção de macronutrientes e supressão do metabolismo respiratório das raízes (KOZLOWSKI, 1997; PEZESHKI, 2001; KREUZWIESER; PAPADOPOULOU; RENNENBERG, 2004). O estresse imposto pela saturação hídrica do solo possui um caráter fortemente seletivo. No decorrer do processo evolutivo, espécies tolerantes desenvolveram uma variedade de estratégias que as capacitaram a ocupar áreas sujeitas ao alagamento do solo. Dentre estas estratégias, ocorrem alterações morfoanatômicas que auxiliam a aeração interna da planta (DAVANSO-FABRO et al., 1998; MEDRI et al., 2002), e alterações metabólicas, com diminuição no consumo de energia, ativação de rotas anaeróbias e menor investimento no crescimento (PIMENTA, 1998; MEDRI et al., 2002). Na maioria das espécies, a estratégia de sucesso combina respostas morfológicas, anatômicas e fisiológicas (JOLY, 1991).
Algumas respostas das plantas ao alagamento podem incluir senescência foliar, redução no crescimento de caules e raízes, desenvolvimento de raízes adventícias, formação de aerênquima e hipertrofia de lenticelas (KOZLOWSKI, 1997). O fechamento estomático, danos no fotossistema II e a diminuição na fotossíntese também são respostas comuns à deficiência de oxigênio no solo causada pelo alagamento (KOZLOWSKI; PALLARDY, 1979; PEZESHKI, 1993). Limitações estomáticas e não estomáticas são responsáveis pelo decréscimo na fotossíntese (KOSLOWSKI, 1997). As mudanças na assimilação do carbono podem ser atribuídas a limitações fotossintéticas não relacionadas aos estômatos (HERRERA et al., 2008), como alterações nos pigmentos foliares e na Rubisco (KOZLOWSKI, 1997; PEZESHKI, 2001). Apesar das condições desfavoráveis, muitos estudos mostram que o alagamento não impede completamente a ocorrência de determinados processos fisiológicos. Mesmo alagadas, algumas espécies arbóreas perenes são fotossinteticamente ativas, enquanto outras podem continuar crescendo e produzindo folhas, flores e frutos durante o alagamento (WORBES, 1997; SCHÖNGART et al., 2002).

Aegiphila sellowiana Cham. (Lamiaceae) é uma espécie arbórea, com altura de 4-7 m, conhecida popularmente como tamanqueira ou pau-detamanco. É uma planta decídua, heliófita, pioneira, que cresce muito bem em diferentes tipos de solo, 
sendo característica de formações secundárias de florestas ombrófilas e semideciduais (LORENZI, 2002). A espécie tem sido intensamente utilizada em projetos de recomposição de áreas degradadas, algumas das quais são ribeirinhas e estão sujeitas ao alagamento periódico do solo. O estudo das características adaptativas de espécies tropicais, presentes ao longo de formações ciliares, é importante para elucidar alguns aspectos da sobrevivência em solo alagado. Nesse sentido, o objetivo deste trabalho foi verificar os efeitos do alagamento do solo na sobrevivência, crescimento e no metabolismo de A. sellowiana, procurando responder as seguintes questões: 1) $\mathrm{O}$ alagamento do solo afeta a mortalidade e o crescimento de plantas jovens de $A$. sellowiana? 2) $\mathrm{O}$ alagamento do solo afeta a condutância estomática e a taxa fotossintética de plantas jovens de A. sellowiana? 3) A espécie é tolerante ao alagamento do solo?

\section{Material e Métodos}

\section{Coleta de sementes e produção de mudas}

Plantas de A. sellowiana foram obtidas a partir de sementes coletadas em diferentes áreas de vegetação secundária, próximas ou distantes de rios, nos municípios de Arapongas (23 23' $\left.55^{\prime \prime} \mathrm{S} ; 51^{\circ} 19^{\prime} 37^{\prime \prime} \mathrm{W}\right)$, Londrina (232 $20^{\prime} 47^{\prime \prime} \mathrm{S}$; $\left.51^{\circ} 11^{\prime} 48^{\prime \prime} \mathrm{W}\right)$, Nova Santa Bárbara (2336' $\left.53^{\prime \prime} \mathrm{S} ; 50^{\circ} 46^{\prime} 25^{\prime \prime} \mathrm{W}\right)$ e São Jerônimo da Serra

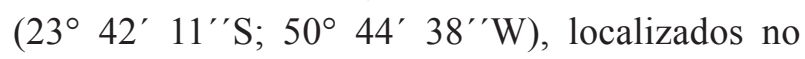
norte do Paraná. Foram coletadas sementes de dois indivíduos de cada local. A germinação ocorreu em tubetes contendo solo constituído de $80 \%$ de terra e $20 \%$ de mistura de palha de grama, cama de frango, calcário e borra de café. Após seis meses, as plantas foram transplantadas para vasos plásticos de $4 \mathrm{~L}$, com solo formado por terra e areia (3:1).

\section{Análises de mortalidade e crescimento}

Para essas análises, foram constituídos quatro grupos de plantas: D (controle; 15 indivíduos) - plantas mantidas em solo drenado; A80 (15 indivíduos) - plantas mantidas em solo alagado por 80 dias; AD (15 indivíduos) - plantas mantidas sob alagamento por 50 dias seguidos de reaeração por 30 dias; $\mathrm{R}$ (referencial; 15 indivíduos) - plantas sacrificadas no primeiro dia do experimento e utilizadas como referência para os experimentos de crescimento. Para os grupos de plantas em condição de alagamento, os vasos foram vedados e mantidos com lâmina d'água a $1,5 \mathrm{~cm}$ acima do solo. O experimento foi inteiramente casualizado, distribuindo-se grupos e plantas por sorteio em bancadas na casa de vegetação. Estes períodos experimentais foram escolhidos porque $A$. sellowiana ocorre naturalmente em áreas do Sul e Sudeste do Brasil, nas quais o período chuvoso concentra-se entre setembro e abril, havendo um pico de chuvas entre dezembro e fevereiro. Desse modo, em algumas áreas ciliares e/ou em depressões do terreno, neste período mais chuvoso, o solo pode permanecer alagado ou encharcado por um período de até 90 dias.

No primeiro dia de montagem do experimento, as plantas do grupo $\mathrm{R}$ foram sacrificadas $\mathrm{e}$ separadas em raízes, caule e folhas, servindo como referencial para as análises de crescimento. Foram tomadas medidas de comprimento de raízes e caule, assim como do diâmetro do colo e caule de cada planta. As medidas de diâmetro do caule foram tomadas a $4 \mathrm{~cm}$ de altura em relação ao colo. Em seguida, raízes, caules e folhas foram desidratados em estufa a $60^{\circ} \mathrm{C}$, por $72 \mathrm{~h}$. Posteriormente, a massa seca foi medida em balança de precisão digital, marca Marte, Modelo AY220. Após os 80 dias de tratamento, as plantas dos grupos D, A80, AD foram submetidas aos mesmos procedimentos 
realizados com o grupo R. A partir desses dados, foi calculada a Taxa de Crescimento Relativo (TCR), utilizando-se a fórmula TCR $=1 n \mathrm{MSf}$ - lnMSi / tempo, onde MSf é a massa seca final e MSi é a massa seca inicial. A TCR expressa o crescimento como uma função do ganho de massa seca com o tempo. As medidas de massa seca, tomadas de todos os grupos, foram utilizadas no cálculo da taxa de crescimento relativo para órgãos individualizados e também para a planta inteira.

Foi realizada, semanalmente, a contagem dos indivíduos mortos e a quantidade de folhas produzidas e perdidas por cada planta, em todos os grupos, durante todo o período experimental. Para tanto, no primeiro dia do experimento, foi feita uma demarcação, utilizando um fio de algodão, imediatamente acima da última folha totalmente expandida. Nessa análise, foram utilizadas apenas as plantas que sobreviveram até o fim do experimento. Ainda foi observada a produção de raízes diageotrópicas. Os dados foram submetidos à análise de variância (ANOVA) e as médias foram comparadas pelo teste de Tukey (AYRES et al., 2005), considerando um nível de significância de $5 \%$.

\section{Análises fisiológicas foliares}

Para estas análises, foram constituídos cinco grupos de plantas: D (controle; 15 indivíduos) plantas mantidas em solo drenado; A4, A9, A13 e A28 (15 indivíduos em cada grupo) - plantas mantidas, respectivamente, em solo alagado por 4, 9, 13 ou 28 dias. O experimento foi inteiramente casualizado, distribuindo-se grupos e plantas por sorteio em bancadas na casa de vegetação.

Foram realizadas medidas da condutância estomática $\left(\mathrm{g}_{\mathrm{s}}\right)$, taxa fotossintética líquida $(A) \mathrm{e}$ concentração de $\mathrm{CO}_{2}$ intercelular, por meio de um analisador de gases por infravermelho, modelo LI-6200 (Li-Cor). Para tanto, foi utilizada sempre uma folha totalmente expandida do terceiro ou quarto nó a partir do ápice de cada planta, com aproximadamente 25 segundos de leitura em cada folha, intensidade luminosa em torno de 1850 4moles $\mathrm{m}^{-2} \cdot \mathrm{s}^{-1}$ e temperatura de $36^{\circ} \mathrm{C}$. Foram utilizadas sete plantas de cada grupo. As avaliações iniciaram-se às nove horas e foram realizadas em sete etapas, tomando-se a cada uma delas, medidas de folhas do controle e de cada um dos tratamentos logo em seguida. Os dados foram submetidos à análise de variância (ANOVA) e as médias foram comparadas pelo teste de Tukey (AYRES et al., 2005), considerando um nível de significância de $5 \%$.

\section{Resultados}

\section{Mortalidade e crescimento}

Nos primeiros dez dias de alagamento, 33,3\% das plantas apresentaram murcha completa, seguida de morte (Tabela 1). Dentre as $66,7 \%$ sobreviventes, o alagamento diminuiu o comprimento de raízes e caules e a TCR de raízes, caule, folhas e plantas inteiras (Tabelas 2 e 3). As plantas alagadas também apresentaram menor diâmetro de caule. Entretanto, em média, o alagamento não afetou o diâmetro do colo (Tabela 2). Mesmo assim, alguns indivíduos alagados apresentaram base do caule bastante hipertrofiada (Figuras 1 a 4). O decréscimo da TCR de folhas foi o que mais contribuiu para a redução na TCR de plantas inteiras (Tabela 3). O alagamento estimulou a produção de raízes diageotrópicas (Tabelas 1; Figuras 1).

A abscisão foi maior nas plantas submetidas a algum período de alagamento do solo, enquanto a produção de folhas novas foi maior nas plantas cultivadas em solo drenado (Tabela 1). As plantas dos grupos A80 e AD produziram raízes diageotrópicas, o que não foi observado nas plantas do grupo D. Nas plantas do grupo AD, após o término do alagamento, ocorreu a recuperação da produção de folhas novas. 
Tabela 1 - Mortalidade, número de folhas que sofreram abscisão e número de folhas novas e raízes diageotrópicas produzidas por planta de Aegiphila sellowiana Cham., em solos drenado (D), alagado por 80 dias (A80) e alagado por 50 dias seguido de 30 dias de drenagem (AD). Para os cálculos, foram consideradas apenas folhas novas e perdidas pelas plantas que sobreviveram. Valores com pelo menos uma mesma letra não diferem estatisticamente pelo Teste de Tukey $(\leq 0,05)$.

\begin{tabular}{lccc}
\hline & D & A80 & AD \\
\hline Mortalidade (\%) & 0 & 33,3 & 33,3 \\
Abscisão & $5,10 \mathrm{~b}$ & $7,70 \mathrm{a}$ & $8,10 \mathrm{a}$ \\
Folhas novas & $7,50 \mathrm{a}$ & $4,10 \mathrm{~b}$ & $7,00 \mathrm{a}$ \\
Raízes diageotrópicas & $0,00 \mathrm{~b}$ & $5,66 \mathrm{a}$ & $3,60 \mathrm{a}$ \\
\hline
\end{tabular}

Fonte: Autores.

Tabela 2 - Morfometria de raízes, colo e caule de Aegiphila sellowiana Cham. mantidas em solos drenado (D), alagado por 80 dias (A80) e alagado por 50 dias seguidos de 30 dias de drenagem (AD). Valores com pelo menos uma mesma letra não diferem estatisticamente pelo Teste de Tukey $(\leq 0,05)$.

\begin{tabular}{lccc}
\hline & D & A80 & AD \\
\hline Comprimento da raiz $(\mathrm{cm})$ & $53,00 \mathrm{a}$ & $34,47 \mathrm{~b}$ & $25,67 \mathrm{c}$ \\
Comprimento do caule $(\mathrm{cm})$ & $27,90 \mathrm{a}$ & $23,02 \mathrm{~b}$ & $25,40 \mathrm{ab}$ \\
Diâmetro do colo $(\mathrm{cm})$ & $0,85 \mathrm{a}$ & $0,80 \mathrm{a}$ & $0,78 \mathrm{a}$ \\
Diâmetro da base do caule $(\mathrm{cm})$ & $0,54 \mathrm{a}$ & $0,44 \mathrm{a}$ & $0,37 \mathrm{a}$ \\
\hline
\end{tabular}

Fonte: Autores.

Tabela 3 - Taxa de Crescimento Relativo (TCR) de raízes, caule, folhas e plantas inteiras de Aegiphila sellowiana Cham. mantidas em solos drenado (D), alagado por 80 dias (A80) e alagado por 50 dias seguidos de 30 dias de drenagem (AD). Valores com pelo menos uma mesma letra não diferem estatisticamente pelo Teste de Tukey $(\leq 0,05)$.

\begin{tabular}{lccc}
\hline & D & A80 & AD \\
\hline Raiz & $16,82 \mathrm{a}$ & $4,95 \mathrm{~b}$ & $4,95 \mathrm{~b}$ \\
Caule & $5,75 \mathrm{a}$ & $1,29 \mathrm{~b}$ & $1,47 \mathrm{~b}$ \\
Folha & $8,53 \mathrm{a}$ & $-4,90 \mathrm{~b}$ & $-0,01 \mathrm{~b}$ \\
Planta & $22,39 \mathrm{a}$ & $12,08 \mathrm{~b}$ & $13,16 \mathrm{~b}$ \\
\hline
\end{tabular}

Fonte: Autores. 
Figuras 1- Aspecto geral da superfície do solo e da base de caules de Aegiphila sellowiana Cham. mantidas em diferentes tratamentos. 1 - Drenado (D), com caule sem hipertrofia e sem raízes superficiais. 2 e 3 Alagado por 80 dias (A80), com hipertrofia basal e muitas raízes diageotrópicas. 4 - Alagado por 50 dias seguido de drenagem por 30 dias (AD), com moderada hipertrofia basal e algumas raízes diageotrópicas. Legenda: bh - base hipertrofiada; rd - raiz diageotrópica.

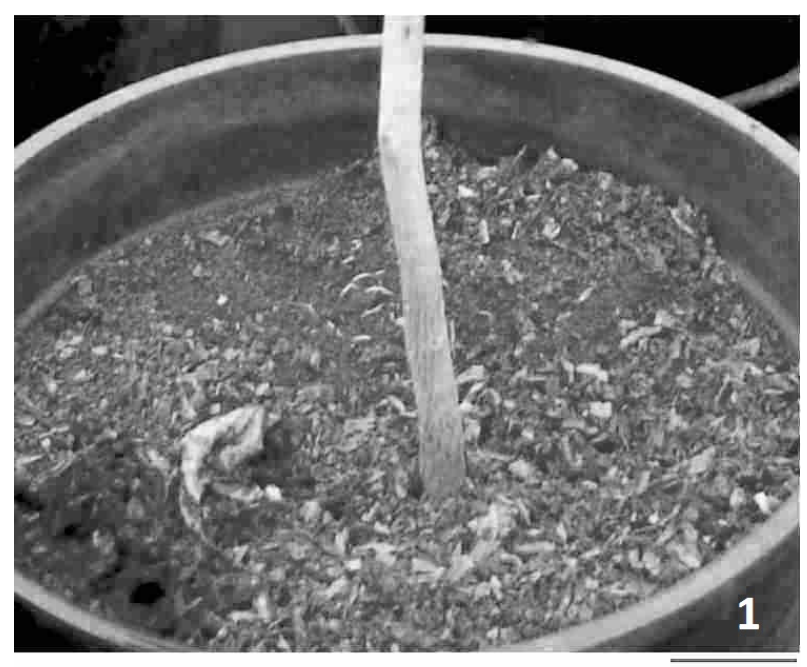

$3,0 \mathrm{~cm}$

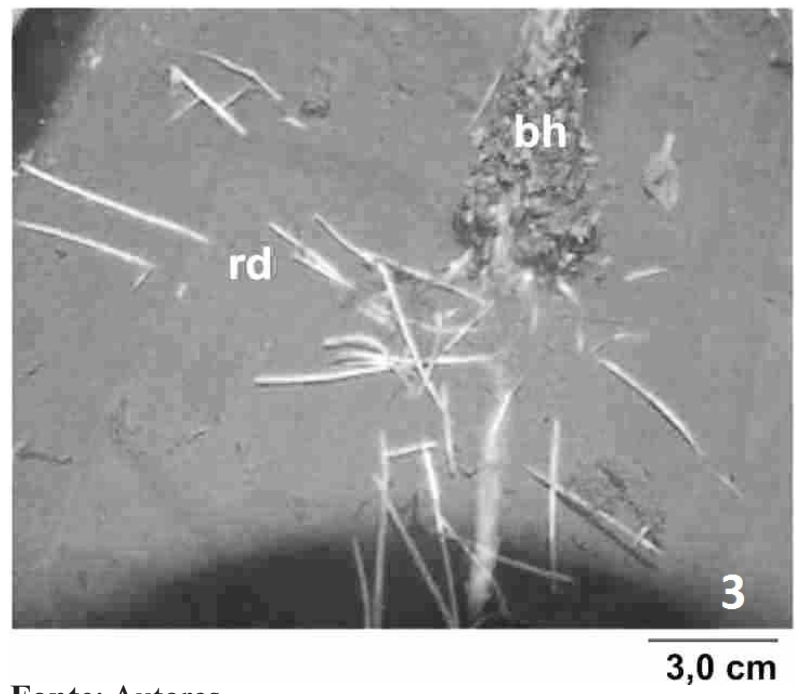

Fonte: Autores.

\section{Respostas fisiológicas foliares}

O alagamento provocou redução na condutância estomática e na taxa fotossintética nos períodos

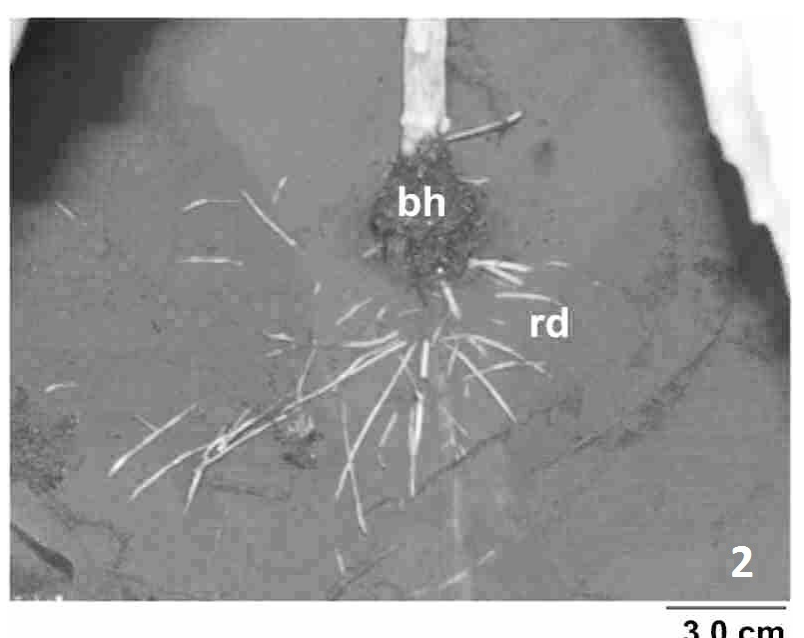

$3,0 \mathrm{~cm}$

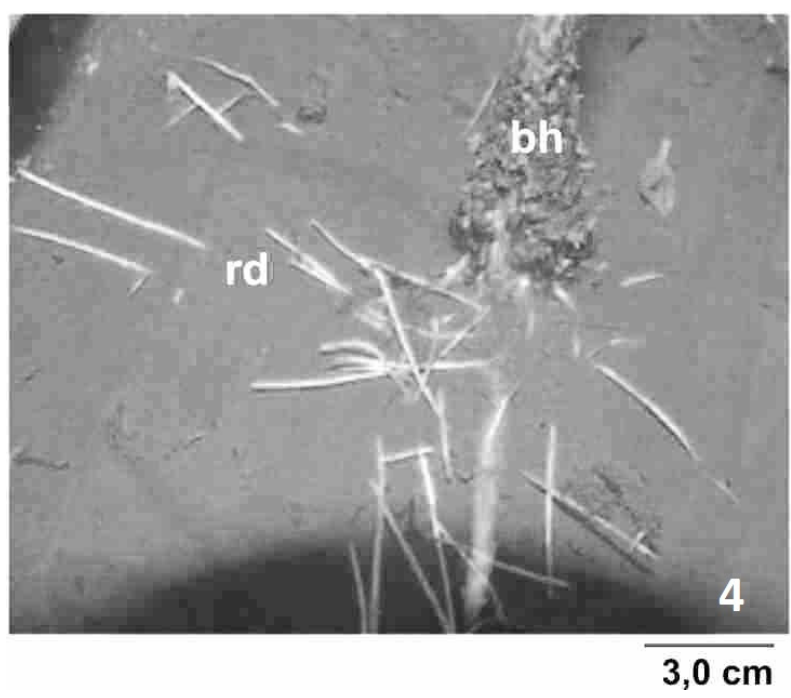

analisados. As plantas do grupo A9 e A13 apresentaram maior concentração intercelular de gás carbônico que as plantas do grupo D (Tabela 4). 
O alagamento do solo afeta a sobrevivência, o crescimento e o metabolismo de Aegiphila sellowiana Cham. (Lamiaceae)?

Tabela 4 - Variáveis fisiológicas foliares em plantas de Aegiphila sellowiana Cham. mantidas em solo drenado (D) ou alagado por 4 (A4), 9 (A9), 13 (A13) ou 28 dias (A28). Valores com pelo menos uma mesma letra não diferem estatisticamente pelo Teste de Tukey $(\leq 0,05)$.

\begin{tabular}{lccccc}
\hline & D & A4 & A9 & A13 & A28 \\
\hline Condutância estomática $\left(\mathrm{mol} \cdot \mathrm{m}^{-} \cdot \mathrm{s}^{-1}\right)$ & $520,00 \mathrm{a}$ & $130,00 \mathrm{~b}$ & $120,00 \mathrm{~b}$ & $90,00 \mathrm{~b}$ & $130,00 \mathrm{~b}$ \\
Taxa fotossintética $\left(\mu \mathrm{mol} \cdot \mathrm{m}^{-2} \cdot \mathrm{s}^{-1}\right)$ & $5,18 \mathrm{a}$ & $0,66 \mathrm{~b}$ & $0,47 \mathrm{~b}$ & $0,00 \mathrm{~b}$ & $0,00 \mathrm{~b}$ \\
Concentração interna de $\mathrm{CO}_{2}$ foliar $\left(\mu \mathrm{mol} \cdot \mathrm{mol}^{-1}\right)$ & $276 \mathrm{~b}$ & $291 \mathrm{ab}$ & $298 \mathrm{a}$ & $307 \mathrm{a}$ & $289 \mathrm{ab}$ \\
\hline
\end{tabular}

Fonte: Autores.

\section{Discussão}

\section{Mortalidade e crescimento}

Os resultados desse estudo mostraram um efeito severo do alagamento do solo sobre a sobrevivência de A. sellowiana. Outras espécies arbóreas neotropicais, quando submetidas ao alagamento, também apresentaram alto percentual de mortalidade, como Schizolobium parahyba (Vell.) S.F. Blake (COSTA et al., 2006) e Caesalpinia peltophoroides Benth. (HENRIQUE et al., 2010), o que explica a baixa frequência das mesmas em planícies de inundação. Por outro lado, Calophyllum brasiliense Camb. (OLIVEIRA; JOLY, 2010), Cecropia pachystachya Trec. (BATISTA et al., 2008) e Sebastiania commersoniana (Baillon) Smith \& Downs (KOLB et al., 1998), espécies típicas de planícies de inundação ou áreas ciliares, não apresentaram mortalidade sob condição de alagamento.

Além da severa mortalidade, os fortes efeitos do alagamento no crescimento e desenvolvimento, observados em A. sellowiana, também foram registrados em outras espécies arbóreas neotropicais, como Jacaranda puberula Cham. (PIMENTA et al., 1996), Peltophorum dubium (Sprengel) (MEDRI et al., 1998), Chorisia speciosa St. Hil. (BIANCHINI et al., 2000), Chrysophyllum gonocarpum (Mart. \& Eichl.) Engl. (BIANCHINI, 1998), Campomanesia xanthocarpa O. Berg. (MEDRI et al., 2002) e Lithraea molleoides (Vell.) Eng. (MEDRI et al., 2007). Efeitos discretos do alagamento do solo sobre o crescimento e desenvolvimento já foram observados em alguns estudos, como em Tabebuia avellanedae Lorentz ex Griseb, onde somente raízes e caule apresentaram diminuição no crescimento (DAVANSO et al., 2002), e em Cecropia pachystachya (BATISTA et al., 2008). No entanto, a maioria das espécies tolerantes neotropicais tem apresentado significativa redução do crescimento da planta como um todo, como foi observado por Kolb et al. (1998) em Sebastiania commersoniana, e por Mielke et al. (2003) em Genipa americana L.

Plantas alagadas de A. sellowiana apresentaram menor TCR e menor comprimento de caules e raízes. A diminuição da TCR radicial ocorreu devido à morte de parte do sistema original de raízes, não sendo suficientemente compensada pela origem de raízes diageotrópicas. Batista et al. (2008) observaram que 30 dias de alagamento causaram a morte de parte do sistema radicial original em Cecropia pachystachya. Entretanto, tal evento foi parcialmente compensado pela formação de novas raízes superficiais. Embora tenha sido observado em Sesbania virgata (Cav.) Pers. (DAVANSO-FABRO et al., 1998), em Cytharexyllum myrianthum Cham. (ANDRADE et al., 1999) e em Annona glabra L. (Mielke et al., 2005), são raras as espécies tolerantes estudadas que não apresentam redução do crescimento das raízes quando submetidas ao alagamento do solo.

Em condição de alagamento, a menor TCR total foi causada pela morte de parte do sistema de raízes, pela maior abscisão foliar e menor produção de novas folhas e pelo menor crescimento do caule. De forma contrastante, sob alagamento, o aumento da 
massa seca tem sido relatado em muitas espécies típicas de ambientes alagados, como Nyssa aquatica L. (MCKEVLIN; HOOK; MCKEE JUNIOR, 1995), Talauma ovata A. St. -Hil. (LOBO; JOLY, 1995) e Sesbania virgata (DAVANSO-FABRO et al., 1998). Mesmo assim, a redução do crescimento não deve ser entendida, isoladamente, como intolerância ao alagamento, pois este processo também foi observado em outras espécies típicas de ambientes alagados, como em Sebastiania commersoniana (KOLB et al., 1998), sendo, muitas vezes, parte da estratégia de tolerância ao estresse gerado pelo alagamento do solo. Em muitas espécies, a diminuição do crescimento de alguns órgãos, durante o alagamento, leva à economia de energia e à manutenção de mínimo funcionamento do metabolismo nas regiões mais afetadas pela hipoxia (WIEDENROTH, 1993; ARMSTRONG; BRAENDLE; JACKSON, 1994). Batista et al. (2008) afirmaram que a diminuição da TCR da raiz e do caule de plantas de Cecropia pachystachya, cultivadas em solo alagado, pode ser uma conseqüência da menor quantidade de energia disponível, já que, devido à hipoxia ocasionada pelo alagamento, as plantas tendem a desviar o metabolismo para vias fermentativas, resultando em uma redução dos níveis energéticos (CRAWFORD; BRAENDLE, 1996).

$\mathrm{O}$ alagamento afetou pouco o comprimento e o diâmetro de caules em A. sellowiana. Porém, a TCR do caule foi significativamente menor que a obtida para o grupo controle. Este resultado corrobora análises teciduais realizadas em plantas alagadas desta espécie (MEDRI et al., 2011), as quais mostraram que tecidos radiciais ou caulinares provenientes de plantas cultivadas em solo alagado têm menor densidade, resultado da presença de células maiores e mais vacuolizadas e de muitos espaços intercelulares. Segundo 7 (1981), o crescimento radial de células do córtex pode levar ao desenvolvimento de grandes espaços de ar (aerênquima), importantes na difusão de oxigênio da parte aérea para as raízes. Estes efeitos podem ser desencadeados pelo etileno, que possui seus níveis aumentados sob condição de hipoxia, como sugerido por Pimenta et al. (1996) e por Medri et al. (1998).

$\mathrm{O}$ efeito do alagamento foi bastante severo em folhas de A. sellowiana, principalmente porque o estresse estimuloua abscisão em todos os tratamentos. A produção de novas folhas foi menor nas plantas alagadas, não sendo suficiente para compensar a perda de massa pela maior abscisão. Pimenta (1998) registrou decréscimo da área foliar total em plantas de Campomanesia xanthocarpa cultivadas em solo alagado, conseqüência da maior abscisão de folhas e menor produção de folhas novas. Nash e Graves (1993), pelos mesmos motivos, verificaram que a hipoxia levou a uma menor área foliar total em Nyssa silvatica Marsh. Segundo Drew (1991), o alagamento reduz a absorção e o transporte de íons, resultando em um estresse mineral para a parte aérea, geralmente, acompanhado de senescência e abscisão prematura de folhas. Pimenta (1998) afirmou que os efeitos do alagamento sobre a abscisão e produção de folhas podem ser reflexos da deficiência nutricional e da queda na condutância estomática e taxa fotossintética.

\section{Respostas fisiológicas foliares}

Em A. sellowiana, possíveis variações nos níveis hormonais provocados pelo alagamento, principalmente o aumento do ácido abscísico (ABA) e queda das citocininas, podem ter provocado o fechamento estomático e estimulado a abscisão, como já observado em outros estudos com plantas em condições de hipoxia (SELMAN; SANDANA, 1972; NEUMAN; ROOD; SMIT, 1990; NEUMAN; SMIT, 1991; ZHANG; ZHANG, 1994). A redução na condutância estomática em plantas alagadas, somada à alta concentração de $\mathrm{CO}_{2}$ encontrada nos espaços intercelulares do mesofilo destas plantas, sugere que o fechamento estomático não foi a única causa de redução da taxa fotossintética. Possivelmente, nesta espécie, o alagamento também deve afetar a atividade da Rubisco ou 
provocar danos ao fotossistema II (LIAO; LIN, 1994; DREYER, 1994; KOZLOWSKI, 1997; PEZESHKI, 2001; HERRERA et al., 2008). Mielke et al. (2003) relataram que, em Genipa americana, a taxa fotossintética foi limitada por fatores estomáticos e não estomáticos em plantas alagadas. Pezeshki (2001) reforçou que, em longos períodos de alagamento, limitações não estomáticas, como a degradação de pigmentos e alterações em enzimas do Ciclo de Calvin são as causas principais do declínio fotossintético.

A queda na taxa fotossintética, com conseqüente queda na produção de fotoassimilados, está relacionada com a queda na TCR verificada em plantas alagadas de A. sellowiana. Em muitas espécies vegetais, a hipoxia pode provocar variações na respiração aeróbia (JOLY, 1994; ROGGE et al., 1998; MATSUI; TSUCHIYA, 2006), no nível nutricional (DREW, 1991; MEDRI et al., 2002; ALAOUI-SOSSÉ et al., 2005) e na fotossíntese (LIAO; LIN, 1996; OLIVELLAet al., 2000; PRYOR; DAVISON; CLOSE, 2006; FERNANDEZ, 2006), e pode afetar o crescimento e desenvolvimento das diferentes partes da planta em ambiente alagado (MEDRI et al., 1998, 2002; DAVANSO et al., 2002; PRYOR; DAVISON; CLOSE, 2006). Desse modo, a sobrevivência da planta é dependente de um balanço na distribuição de fotoassimilados entre as suas várias partes (WIEDENROTH, 1993; ARMSTRONG; BRAENDLE; JACKSON, 1994).

\section{Conclusões}

A morte de 33,3\% das plantas, já nos primeiros dias de alagamento do solo, sugere que A. sellowiana é intolerante ao estresse causado por esta variação ambiental. Entretanto, 66,7\% das plantas submetidas a tratamentos com diferentes períodos de alagamento sobreviveram e, apesar de apresentarem desenvolvimento limitado, com abscisão foliar, baixa produção de novas folhas e queda na condutância estomática e fotossíntese, produziram respostas típicas de plantas tolerantes, como o desenvolvimento de raízes diageotrópicas e queda na TCR, e isso pode ser vista como uma forma de economia de energia e sobrevivência. Estes resultados sugerem que A. sellowiana, numa análise global para a espécie, pode ser considerada intolerante ao alagamento. Porém, no conjunto de populações distribuídas por sua área de ocorrência, pode haver genótipos que conferem diferentes características relacionadas a uma menor ou maior tolerância ao alagamento do solo, os quais podem ter sido selecionados para utilização na experimentação. Esta hipótese é reforçada pelo delineamento de coleta de sementes para obtenção de mudas, realizado neste trabalho, o qual privilegiou a obtenção de matrizes de diferentes locais e populações, o que, em teoria, maximiza a variabilidade genética presente no conjunto de sementes obtido. De qualquer modo, a variável genética também deve ser levada em conta em futuros trabalhos, buscando entender de que forma a diversidade genética dentro da espécie pode influenciar a tolerância da mesma ao estresse provocado pelo alagamento do solo.

\section{Agradecimentos}

Os autores agradecem a CAPES/CNPq, pelo apoio financeiro fornecido através de bolsas de estudo. Agradecem, ainda, ao Programa de PósGraduação em Ecologia de Ambientes Aquáticos Continentais (PEA), pelo apoio financeiro e institucional.

\section{Referências}

ALAOUI-SOSSÉ, B.; GÉRARD, B.; TOUSSAINT, M.; BADOT, P. Influence of flooding on growth, nitrogen availability in soil, and nitrate reduction of young oak seedlings (Quercus robur L.). Annals of Forest Science, Champenoux, v. 62, p. 593-600. 2005.

ANDRADE, A. C. S.; RAMOS, F. N.; SOUZA, A. F.; LOUREIRO, M. B.; BASTOS, R. Flooding effects of Cytharexyllum myrianthum Cham. and Genipa americana L.: responses of two neotropical lowland species. Revista Brasileira de Botânica, São Paulo, v. 22, n. 2, p. 281-285. 1999. 
ARMSTRONG, W.; BRAENDLE, R.; JACKSON, M. B. Mechanisms of flood tolerance in plants. Acta Botanica Neerlandica, Amsterdam, v. 43, n. 4, p. 307-358. 1994.

AYRES, M.; AYRES JUNIOR, M.; AYRES, D. L.; SANTOS, A. S. Bioestat: 2.0: aplicações estatísticas nas áreas das ciências biológicas e médicas. Belém: Sociedade Civil Mamirauá, 2005.

BATISTA, C. U. N.; MEDRI, M. E.; BIANCHINI, E.; MEDRI, C.; PIMENTA, J. A. Tolerância à inundação de Cecropia pachystachya Trec. (Cecropiaceae): aspectos ecofisiológicos e morfoanatômicos. Acta Botanica Brasilica, Feira de Santana, v. 22, n. 1, p. 91-98. 2008.

BIANCHINI, E. Ecologia de população de Chrysophyllum gonocarpum ( Mart. \& Eichler) Engl. no Parque Estadual Mata dos Godoy, Londrina, PR. 1998. 165 f. Tese (Doutorado em Ciências Biológicas) - Universidade Estadual de Campinas, Campinas.

BIANCHINI, E.; MEDRI, M. E.; PIMENTA, J. A.; GILONI, P. C.; KOLB, R. M.; CORREA, G. T. Anatomical alterations in plants of Chorisia speciosa A. St- Hil. submitted to flooding. Interciencia, Caracas, v. 25, n. 9, p. 436-441. 2000.

COSTA, A. M.; GOBBI, E. L.; DEMUNER, V. G.; HEBLING, S. A. O efeito da inundação do solo sobre o crescimento inicial de Schizolobium parahyba (Vell.) S.F. Blake, guapuruvu. Natureza on line, Santa Tereza, v. 4, n. 1, p. 7-13, 2006.

CRAWFORD, R. M. M.; BRAENDLE, R. Oxygen deprivation stress in a changing environment. Journal of Experimental Botany, Lancaster, v. 47, n. 295, p. 145159. 1996.

DAVANSO, V. M.; SOUZA, L. A.; MEDRI, M. E.; PIMENTA, J. A.; BIANCHINI, E. Photosynthesis, growth and development of Tabebuia avellanedae Lor. ex Griseb. (Bignoniaceae) in flooded soil. Brazilian Archives of Biology and Technology, Curitiba, v. 45, n. 3, p. 375-384. 2002.

DAVANSO-FABRO, V.M.; MEDRI, M.E.; BIANCHINI, E.; PIMENTA, J. A. Tolerância à inundação: aspectos da anatomia ecológica e do desenvolvimento da Sesbania virgata (CAV.) Pers. (Fabaceae). Brazilian Archives of Biology and Technology, Curitiba, v. 41 n. 4, p. 475-482. 1998.

DREW, M. C. Oxygen deficiency in the root environment and plant mineral nutrition. In: JACKSON, M. B.; LAMBERS, D. D. (Ed.). Plant life under deprivation. The Hague: SPB Academic Publishing, 1991. p. 303-316.

DREYER, E. Compared sensitivity of seedlings from 3 woody especies (Quercus rubor L., Quercus rubra L. and
Fagus silvatica L.) to water-logging and associated root hypoxia: effects on eater relations and photosynthesis. Annals of Forest Science, Champenoux, v. 51, p. 417429, 1994.

FERNANDEZ, M. D. Changes in photosynthesis and fluorescence in response to flooding in emerged and submerged leaves of Pouteria orinocoensis. Photosynthetica, Olomouc, v. 44, n. 1, p. 32-38. 2006.

HENRIQUE, P. C.; ALVES, J. D.; GOULART, P. F. P.; DEUNER, S.; SILVEIRA, N. M.; ZANANDREA, L.; CASTRO, E. V.; Características fisiológicas e anatômicas de plantas de sibipiruna submetidas à hipoxia. Ciência Rural, Santa Maria, v. 40, n. 1, p. 70-76, 2010.

HERRERA, A.; TEZARA, W.; MARÍN, O.; RENGIFO, E. Stomatal and non-stomatal limitations of photosynthesis in trees of a tropical seasonally flooded forest. Physiologia Plantarum, Lund, v. 134, n. 1, p. 4148, 2008 .

JOLY, C. A. Flooding tolerance in tropical trees. In: JACKSON, M. B.; DAVIES, D. D.; LAMBERS, H. (Ed.). Plant life under oxygen deprivation: ecology, physiology and biochemistry. The Hague: SBP Academic Publishing, 1991. p. 23-34.

JOLY, C. A. Flooding tolerance: a reinterpretation of Crawford's metabolic theory. Proceedings of the Royal Society of Edinburgh, Edinburgh, v. 102 n. B, p. 343-354, 1994.

JUNK, W. J. Wetlands of tropical South America. In: WHIGHAM, D.; HEJNÝ, S.; DYKYJOVÁ, D. (Ed.). Wetlands of the world: inventory, ecology and management. Boston: Dr. W. Junk Publishing, 1993. p. 679-739.

KAWASE, M. Anatomical and morphological adaptation of plants to waterlogging. Horticultural Science, Praha, v. 16 , n. 1, p. 30-34. 1981.

KOLB, R. M.; MEDRI, M. E.; BIANCHINI, E.; PIMENTA, J. A.; GILONI, P. C.; CORREA, G. T. Anatomia ecológica de Sebastiania commersoniana (Baillon) Smith \& Downs (Euphorbiaceae) submetida ao alagamento. Revista Brasileira de Botânica, São Paulo, v. 21, n. 3, p. 305-312. 1998.

KOZLOWSKI, T. T. Responses of woody plants to flooding and salinity. Tree physiology monograph no. 1. Victoria: Heron Publishing, 1997. p. 1-29.

KOZLOWSKI, T. T.; PALLARDY, S. G. Stomatal response of Fraxinus pennsylvanica seedlings during and after flooding. Physiologia Plantarum, Lund, v. 46, n. 2, p. $155-158,1979$. 
KREUZWIESER, J.; PAPADOPOULOU, E.; RENNENBERG, $\mathrm{H}$. Interaction of flooding with carbon metabolism of forest trees. Plant Biology, Freiburg, v. 6, n. 3, p. 299-306, 2004.

LIAO, C. T.; LIN, C. H. Effect of flooding stress on photosynthetic activities of Momordica charantia. Plant Physiology and Biochemistry, Paris, v. 32, n. 4, p. 479485. 1994.

LIAO, C. T.; LIN, C. H. Photosynthetic responses of grafted bitter melon seedlings to flood stress. Environmental and Experimental Botany, Paris, v. 36, n. 2, p. 167-172. 1996.

LOBO, P. C.; JOLY, C. A. Mecanismos de tolerância à inundação de plantas de Talauma ovata St. Hill. (Magnoliaceae), uma espécie típica de matas de brejo. Revista Brasileira de Botânica, São Paulo, v. 18, p. 177183, 1995.

LORENZI, H. Árvores brasileiras: manual de identificação e cultivo de plantas arbóreas nativas do Brasil. 4. ed. Nova Odessa: Instituto Plantarum, 2002. v. 1.

MATSUI, T.; TSUCHIYA, T. Root aerobic respiration and growth characteristics of three Typha species in response to hypoxia. Ecological Research, Tokyo, v. 21, n. 3, p. 470-475. 2006.

MCKEVLIN, M. R.; HOOK, D. D.; MCKEE JUNIOR, W. H. Growth and nutrient use efficiency of water tupelo seedlings in flooded and well-drained soil. Tree Physiology, Oxford, v. 15, n. 11, p. 753-758. 1995.

MEDRI, C.; MEDRI, M. E.; RUAS, E. A.; SOUZA, L. A.; MEDRI, P. S.; SAYHUN, S.; BIANCHINI, E.; PIMENTA, J. A. Morfoanatomia de órgãos vegetativos de plantas juvenis de Aegiphila sellowiana Cham. (Lamiaceae) submetidas ao alagamento do substrato. Acta Botanica Brasilica, Feira de Santana, v. 25, n. 2, p. 445-454, 2011.

MEDRI, M. E.; BIANCHINI, E.; PIMENTA, J. A.; COLLI, S.; MULLER, C. Estudos sobre a tolerância ao alagamento em espécies arbóreas nativas da bacia do rio Tibagi. In: MEDRI, M. E.; BIANCHINI, E.; SHIBATTA, O. A.; PIMENTA, J. A. (Ed.). A bacia do Rio Tibagi. Londrina: Edição dos editores, 2002. p. 133-172.

MEDRI, M. E.; BIANCHINI, E.; PIMENTA, J. A.; DELGADO, M. T.; CORREA, G. T. Aspectos morfoanatômicos e fisiológicos de Peltophorum dubium (Spr.) Taub. submetida ao alagamento e aplicação de ethrel. Revista Brasileira de Botânica, São Paulo, v. 21, n. 3, p. 261-267, 1998.

MEDRI, M. E.; FERREIRA, A. C.; KOLB, R. M.; BIANCHINI, E.; PIMENTA, J. A.; DAVANSO-FABRO,
V. M.; MEDRI, C. Alterações morfoanatômicas em plantas de Lithraea molleoides (Vell.) Engl. submetidas ao alagamento. Acta Scientiarum Biological Sciences, Maringá, v. 29, n. 1, p. 15-22, 2007.

MIELKE, M. S.; ALMEIDA, A. F.; GOMES, F. P.; AGUILAR, M. A. G.; MANGUABEIRA, P. A. O. Leaf gas exchange, chlorophyll fluorescence and growth responses of Genipa americana seedlings to soil flooding. Environmental and Experimental Botany, Paris, v. 50, n. 3, p. 221-231, 2003.

MIELKE, M. S.; MATOS, E. M.; COUTO, V. B.; ALMEIDA, A. F.; GOMES, F. B.; MANGABEIRA, P. A. O. Some photosynthetic and growth responses of Annona glabra L. seedlings to soil flooding. Acta Botanica Brasilica, Feira de Santana, v. 19, n. 4, p. 905-911. 2005.

NASH, L. J.; GRAVES, W. R. Drought and flood stress effects on plant development and leaf water relations of five taxa of trees native to bottomland habitats. Journal of the American Society for Horticultural Science, Alexandria, v. 118, n. 6, p. 845-850. 1993.

NEUMAN, D. S.; ROOD, S. B.; SMIT, B. A. Does cytokinin trasport from root to shoot in the xylem sap regulate leaf responses to root hypoxia? Journal of Experimental Botany, Lancaster, v. 41, n. 10, p. 13251333, 1990.

NEUMAN, D. S.; SMIT, B. A. The influence of leaf water status and ABA on leaf growth and stomata of Phaseolus seedlings with hypoxic roots. Journal of Experimental Botany, Lancaster, v. 42, n. 12, p. 1499-1506, 1991.

OLIVEIRA, V. C.; JOLY, C. A. Flooding tolerance of Callophyllum brasiliense Camb. (Clusiaceae): morphological, physiological and growth responses. Trees, Vancouver, v. 24, p. 185-193, 2010.

OLIVELLA, C.; BIEL, C.; VENDRELL, M.; SAVE, R. Hormonal and physiological responses of Gerbera jamesonii to flooding stress. Horticultural Science, Praha, v. 35 , n. 2, p. 222-225, 2000.

PEZESHKI, S. R. Differences in patterns of photosynthetic responses to hypoxia in flood tolerant and flood-sensitive tree species. Photosynthetica, Olomouc, v. 28, n. 3, p. 423-430, 1993.

PEZESHKI, S. R. Wetland plant responses to soil flooding. Environmental and Experimental Botany, Paris, v. 46, n. 3, p. 299-312, 2001.

PIMENTA, J. A. Estudo populacional de Campomanesia xanthocarpa O. Berg (Myrtaceae) no Parque Estadual Mata dos Godoy, Londrina, PR. 1998. 150 f. Tese (Doutorado em Ciências Biológicas) - Universidade Estadual de Campinas, Campinas. 
PIMENTA, J. A.; BIANCHINI, E.; MEDRI, M. E.; MULLER, C.; OKAMOTO, J. M.; FRANCISCONI, L. M. J.; CORREA, G. T. Aspectos da morfo-anatomia e fisiologia de Jacaranda puberula Cham. (Bignoniaceae) em condições de hipoxia. Revista Brasileira de Botânica, São Paulo, v. 19, n. 2, p. 215-220, 1996.

PRYOR, R. J.; DAVISON, N. J.; CLOSE, D. C. Waterlogging duration; Interspecific comparison of Leptospermum scoparium (Forst et Forst.f.), Acacia melanoxylon (R. Br.), Nothofagus cunninghamii (Hook.) and Eucalyptus abliqua (L'Herit). Austral Ecology, Adelaide, v. 31, n. 4, p. 408-416, 2006.

ROGGE, G. D.; PIMENTA, J. A.; BIANCHINI, E.; MEDRI, M. E.; DOS ANJOS, S. C.; ALVES, L. M. T. Metabolismo respiratório de raízes de espécies arbóreas tropicais submetidas à inundação. Revista Brasileira de Botânica, São Paulo, v. 21, n. 2, p. 153-158, 1998.

SCHÖNGART, J.; PIEDADE, M. T. F.; LUDWIGSHAUSEN, S.; HORNAS, V.; WORBES, M. Phenology and stem-growth periodicity of tree species in Amazonian floodplain forests. Journal of Tropical Ecology, Cambridge, v. 18, n. 1, p. 581-597, 2002.

SELMAN, I. W.; SANDANA, S. Growth responses of tomato plants in non-aerated water culture to foliar sprays of gibberellic acid and benzyladenine. Annals of Botany, Oxford, v. 36, n. 4, p. 837-848, 1972.

WIEDENROTH, E. M. Responses of roots to hypoxia: their structural and energy relations with the whole plant. Environmental and Experimental Botany, Paris, v. 33, n. 1, p. 45-51, 1993.

WORBES, M. The forest ecosystem of the floodplains. In: JUNK, W. J. (Ed.). The Central Amazon floodplain: Ecology of a pulsing system. Heidelberg: Springer Verlag, 1997, p. 223-266.

ZHANG, J.; ZHANG, X. Can early wilting of old leaves account for much of the ABA accumulation in flooded pea plants? Journal of Experimental Botany, Lancaster, v. 45 , n. 9, p. 1335-1342, 1994.

Recebido em 14 de novembro de 2011 Aceito em 21 de dezembro de 2011 